\title{
Hypertension Associated with Poor Sleep Quality in The Elderly Population in Batu City
}

\author{
Siti Roziah Ria Famuji ${ }^{1}$, Abdul Malik Setiawan ${ }^{2}$, Achdiat Agoes ${ }^{2}$ \\ 1) Faculty of Medicine, UIN Maulana Malik Ibrahim Malang, Indonesia \\ 2) Department of Biomedicine, Faculty of Medicine, UIN Malang, Indonesia \\ riafamuji@gmail.com \\ DOI: http://doi.org/10.29080/jhsp.v5i2.458
}

Received : February 2021, Accepted : July 2021, Published : September, 2021

\section{Keywords}

Sleep quality;

Blood pressure

value;

Elderly

\begin{abstract}
Hypertension affects approximately $26 \%$ of the adult population and it is a leading cause of death in up to $13.5 \%$ worldwide. Hypertension is a disease with the highest prevalence in Indonesia, so good treatment and prevention are needed. Several studies suggest that there is a correlation between hypertension risk factors and the disturbance of sleep quality. Therefore, it is necessary to develop preventive and promotive efforts to obtain optimum blood pressure in patients with hypertension to avoid complications or even death. This study aims to find out the correlation between sleep quality and the value of blood pressure in the elderly $\geq 60$ years old in Batu City. A cross-sectional study was conducted in Batu City in 2020. Stratified random sampling was performed to select the respondents. A validated PSQI questionnaire and sphygmomanometer were used to assess sleep quality and blood pressure, respectively. A Chi-Square test was used to test the hypothesis. There were 391 respondents involved in this study. Most of the respondents have poor sleep quality, 205 respondents $(52,43 \%)$ of which 41 respondents (20\%) have normal blood pressure, and 164 respondents (80\%) have high blood pressure. The statistical analysis shows a significant correlation $(p=0,000)$ between sleep quality and blood pressure. In conclusion, hypertension is associated with poor sleep quality in the geriatric population in Batu City.
\end{abstract}

\section{Introduction}

Hypertension affects approximately $26 \%$ of adults worldwide and it is the leading cause of death up to $13.5 \%$ (1). Hypertension is still a major challenge in Indonesia because this condition is often found in primary health services with a high prevalence, which is $25.8 \%$ (2). The results of the Basic Health Research (Riskesdas) in 2013 showed that hypertension was ranked number one with a percentage of $45.9 \%$ at the elderly 55-64 years old and increasing with age. Elderly with 65-74 years old (57.6\%) and increasing at the age of 75 years (63.8\%) (3). This situation increased significantly in 2018 at elderly 5564 years old (55.2\%). Elderly with 65-74 years old (63.2\%) and increasing at the elderly with 75 years old that is equal to $69.5 \%$ (4). Hypertension is also the number one disease in Batu City, with 10,110 cases in 2018 (5).

From the data mentioned above, it can be seen that with increasing age, the physiological function of the human body decreases due to the ageing process, and the high morbidity rate due to hypertension indicates the presence of multiple morbidities in the elderly (5). Hypertension is also the biggest risk factor for cardiovascular disease which is the number one cause of mortality in the elderly in Indonesia (6). Therefore, prevention related to increasing the number of hypertension is very necessary. The elderly in Indonesia have a percentage of sleep disorders in the form of insomnia as much as $60 \%$ (7). Poor sleep quality correlates with a risk factor for hypertension (8). It has also been shown that lack of sleep duration can increase blood pressure (9). The results of the study which showed a correlation between poor sleep 
quality and hypertension were in line with that carried out by Lu in 2015 which showed an additive interaction between poor sleep quality and short sleep duration on the prevalence of hypertension in adult men (10). Likewise, a study conducted by Hanus in 2015 showed that individuals with hypertension had a negative correlation with sleep quality in adults (8).

This is in contrast to research conducted by Mohani in 2018 which stated that there was no correlation between sleep quality and systolic and diastolic blood pressure in the elderly (11). Likewise, Sforza's research in 2014 denied a correlation between the poor sleep duration and quality in the elderly and the increased prevalence of hypertension (12).

Although there have been many studies that discuss sleep quality, there is no research that looks for the correlation between hypertension and sleeps quality in the elderly 60 years. Therefore, this study aims to explain the correlation between sleep quality and blood pressure values in the elderly age group 60 y.o in Batu City.

\section{Methods}

This study uses a cross-sectional design which was carried out in Batu City in January - March 2020. The total population in Batu City is 217,454 people. The data is an accumulation of 3 sub-districts in Batu City, as many as 54,668 people in Batu District, 99,636 people in Batu District and 63,150 people in Bumiaji District. The study design also refers to the STROBE guidelines for cross-sectional studies in epidemiological studies.

There are independent variables and dependent variables in this study. The dependent variable is the sleep quality of the elderly in Batu City which is measured using the PSQI questionnaire. The independent variable is the blood pressure value measured using medical records and a sphygmomanometer. The data that has been collected was analyzed by using the contingency coefficient test to see the correlation between the independent variable and the dependent variable.

The population of this study is the elderly with the age of 60 years in Batu City with a total of 24,945 people. The sampling technique used stratified random sampling technique starting from normotension, hypertension stage 1 , hypertension stage 2 to hypertension stage 3 . To get a margin of error of $5 \%$, confidence interval of $95 \%$ and response distribution of 50\%, 391 respondents were recruited. Respondents are residents of 3 sub-districts in Batu City with an age of 60 years. All respondents can interact with researchers and are willing to participate in research through written consent in the form of informed consent.

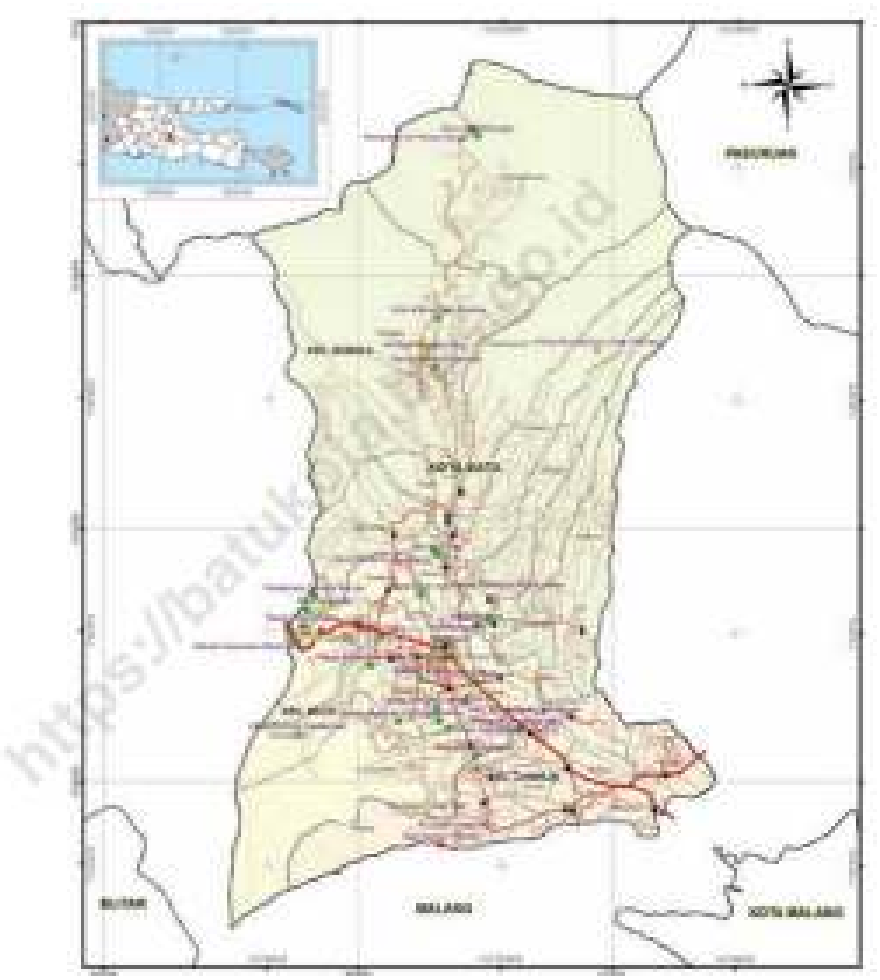

Figure 1 Map of Batu City

Instruments to facilitate data collection, a research questionnaire has been prepared to contain questions about sociodemographic data, history of blood pressure disease and seven sleep quality indicators that assess sleep quality based on a validated PSQI questionnaire. A questionnaire based on the 
Pittsburgh Sleep Quality Index (PSQI) was used to collect sleep quality data for respondents covering seven indicators including subjective sleep quality, sleep latency, sleep duration, sleep disturbances, the efficiency of sleep habits, use of sleeping pills and daytime sleep dysfunction. Blood pressure diagnosis was taken from medical records at the puskesmas and validated by measuring the respondent's blood pressure during sampling. Blood pressure measurement using a calibrated sphygmomanometer was repeated twice.

Data collection was carried out directly by interviewing and providing questionnaires that had been prepared to be filled out by respondents. The researcher explained in advance the benefits and uses of the research. Respondents who agreed to participate in the study were then asked to sign an informed consent. For respondents who cannot read and write, the researcher helps explain the questions and fill out the questionnaire. In data collection, respondents did not receive any gifts from the researchers to be willing to participate in the study.

The answers to questions related to the seven sleep quality indicators according to the PSQI were then tabulated to calculate scores and grouped each sample into good sleep quality (score 5) and poor (score $>5$ ). To determine the correlation between blood pressure and sleep quality, the Chi-Square test was performed. Variables that do not meet the requirements of the Chi-Square test are then tested using the Kruskal-Wallis test. Furthermore, to determine the role of socio-demographic factors, a logistic regression test was carried out using the backward method. The ethical feasibility of this study has been approved by the ethics committee of UIN Maulana Malik Ibrahim Malang and the Ministry of Health of Batu City.

\section{Results}

This study was followed by as many as 391 respondents. The respondents came from Junrejo District as many as 197 respondents, 88 respondents from Batu District and 106 respondents from Bumiaji District. Respondents received assistance in the form of explanations from researchers in filling out questionnaires, to make it easier to understand questions. Data from all respondents have met the requirements for analysis. In this study, there were 336 respondents (85.93\%) female and 55 respondents $(14.07 \%)$ male. When viewed by age, the majority of respondents were aged 60-69 years as many as 235 respondents (60.10\%), followed by age $70-79$ years as many as 122 respondents $(31.20 \%)$, then usia80 years old as many as 34 respondents $(8,70 \%)$.

Table 1 explains that the respondents' last education was dominated by elementary school education (SD) as many as 256 respondents (65.47\%), then the elderly who did not go to school were 51 respondents (13.04\%), junior high school as many as 41 respondents $(10.49 \%)$, SMA 25 respondents (6.40\%), S1 as many as 16 respondents (4.09\%) and S2 as many as 2 respondents (0.51\%). For income per month, namely the group with income $<3,000,000,000$ as many as 351 respondents $(89.77 \%)$ and $>3,000,000,000$ as many as 40 respondents (10.23\%).

When viewed from a family history of hypertension, 219 respondents $(56.01 \%)$ had a family history of hypertension, and the remaining 172 respondents (43.99\%) did not have a family history. The highest body mass index was the normal group as many as 201 respondents (51.41\%). Followed by respondents who are overweight, obesity grade 1 , underweight, obesity grade 2 and obesity grade 3 (27.62\%; 9.97\%; 9.46\%; $1.02 \%$ and $0.51 \%)$. A total of 274 respondents $(70.08 \%)$ did not have the habit of consuming coffee, cigarettes and alcohol. A total of 108 respondents (27.62\%) have a habit of drinking coffee, 5 respondents $(1.28 \%)$ have a smoking habit, 4 respondents $(1.02 \%)$ have a habit of consuming coffee and cigarettes. There are no respondents who have a habit of consuming alcohol.

When viewed from the history of hypertension in the respondents, as many as 219 respondents (56.01\%) had a history of hypertension, while those who did not have a history of hypertension were 172 respondents (43.99\%). A total of 161 respondents $(41.18 \%)$ had a history of taking hypertension drugs, and 230 respondents $(58,82 \%)$. The group that did not suffer from hypertension was 225 respondents $(57.54 \%)$. The group with the most history of hypertension was with hypertension 1-4 years old as many as 76 respondents (19.44\%), then group with hypertension 5-9 years old namely 45 respondents (11.51\%), group with hypertension < 1-year-old as many as 27 respondents $(6.91 \%)$ and the last group is the old group of hypertension 10 years and over, namely 18 respondents $(4.60 \%)$. The characteristics of the respondents based on the demographic data are under table 1 below. 
Table 1 Characteristics of Respondents ( $\mathrm{n}=391)$

\begin{tabular}{|c|c|c|c|c|c|c|c|}
\hline \multirow[t]{2}{*}{ Characteristics } & \multirow[t]{2}{*}{ n (\%) } & \multicolumn{2}{|c|}{$\begin{array}{c}\text { Sleep Quality } \\
\text { n (\%) }\end{array}$} & \multicolumn{4}{|c|}{ Blood Pressure Value (\%) } \\
\hline & & Good & Poor & $\mathbf{N}$ & 1 & 2 & 3 \\
\hline \multicolumn{8}{|l|}{ Gender } \\
\hline Male & $\begin{array}{c}55 \\
(14,07)\end{array}$ & $\begin{array}{c}24 \\
(6,14)\end{array}$ & $\begin{array}{c}31 \\
(7,93)\end{array}$ & $\begin{array}{c}22 \\
(5,63)\end{array}$ & $14(3,58)$ & $\begin{array}{c}12 \\
(3,07)\end{array}$ & $\begin{array}{c}7 \\
(1,79)\end{array}$ \\
\hline Female & $\begin{array}{c}336 \\
(85,93)\end{array}$ & $\begin{array}{c}162 \\
(41,43)\end{array}$ & $\begin{array}{c}174 \\
(44,50)\end{array}$ & $\begin{array}{c}121 \\
(30,95)\end{array}$ & $\begin{array}{c}120 \\
(30,69)\end{array}$ & $\begin{array}{c}72 \\
(18,41)\end{array}$ & $\begin{array}{c}23 \\
(5,88)\end{array}$ \\
\hline \multicolumn{8}{|l|}{ Age } \\
\hline $60-69$ у.о & $\begin{array}{c}235 \\
(60,10)\end{array}$ & $\begin{array}{c}103 \\
(26,34)\end{array}$ & $\begin{array}{c}132 \\
(33,76)\end{array}$ & $\begin{array}{c}85 \\
(21,73)\end{array}$ & $\begin{array}{c}86 \\
(21,99)\end{array}$ & $\begin{array}{c}47 \\
(12,02)\end{array}$ & $\begin{array}{c}17 \\
(4,35)\end{array}$ \\
\hline $70-79$ у.о & $\begin{array}{c}122 \\
(31,20)\end{array}$ & $\begin{array}{c}67 \\
(17,14)\end{array}$ & $\begin{array}{c}55 \\
(14,07)\end{array}$ & $\begin{array}{c}46 \\
(11,76)\end{array}$ & $\begin{array}{c}41 \\
(10,49)\end{array}$ & $\begin{array}{c}26 \\
(6,65)\end{array}$ & $\begin{array}{c}9 \\
(2,30)\end{array}$ \\
\hline$\geq 80$ у.о & $\begin{array}{c}34 \\
(8,70)\end{array}$ & $\begin{array}{c}16 \\
(4,09)\end{array}$ & $\begin{array}{c}18 \\
(4,60)\end{array}$ & $\begin{array}{c}12 \\
(3,07)\end{array}$ & $\begin{array}{c}7 \\
(1,79)\end{array}$ & $\begin{array}{c}11 \\
(2,81)\end{array}$ & $\begin{array}{c}4 \\
(1,02)\end{array}$ \\
\hline \multicolumn{8}{|l|}{ Level of education } \\
\hline No & $\begin{array}{c}51 \\
(13,04)\end{array}$ & $\begin{array}{c}19 \\
(4,86)\end{array}$ & $\begin{array}{c}32 \\
(8,18)\end{array}$ & $\begin{array}{c}15 \\
(3,84)\end{array}$ & $16(4,09)$ & $\begin{array}{c}14 \\
(3,58)\end{array}$ & $\begin{array}{c}6 \\
(1,53)\end{array}$ \\
\hline $\begin{array}{l}\text { Elementary } \\
\text { school }\end{array}$ & $\begin{array}{c}256 \\
(65,47)\end{array}$ & $\begin{array}{c}115 \\
(29,41)\end{array}$ & $\begin{array}{c}141 \\
(36,06)\end{array}$ & $\begin{array}{c}83 \\
(21,23)\end{array}$ & $\begin{array}{c}101 \\
(25,83)\end{array}$ & $\begin{array}{c}53 \\
(13,55)\end{array}$ & $\begin{array}{c}19 \\
(4,86)\end{array}$ \\
\hline $\begin{array}{l}\text { Junior high } \\
\text { school }\end{array}$ & $\begin{array}{c}41 \\
(10,49)\end{array}$ & $\begin{array}{c}22 \\
(5,63)\end{array}$ & $\begin{array}{c}19 \\
(4,86)\end{array}$ & $\begin{array}{c}19 \\
(4,86)\end{array}$ & $\begin{array}{c}9 \\
(2,30)\end{array}$ & $\begin{array}{c}10 \\
(2,56)\end{array}$ & $\begin{array}{c}3 \\
(0,77)\end{array}$ \\
\hline $\begin{array}{l}\text { Senior high } \\
\text { school }\end{array}$ & $\begin{array}{c}25 \\
(6,40)\end{array}$ & $\begin{array}{c}16 \\
(4,09)\end{array}$ & $\begin{array}{c}9 \\
(2,30)\end{array}$ & $\begin{array}{c}14 \\
(3,58)\end{array}$ & $\begin{array}{c}4 \\
(1,02)\end{array}$ & $\begin{array}{c}6 \\
(1,53)\end{array}$ & $\begin{array}{c}1 \\
(0,26)\end{array}$ \\
\hline Diploma/S1 & $\begin{array}{c}16 \\
(4,09)\end{array}$ & $\begin{array}{c}12 \\
(3,07)\end{array}$ & $\begin{array}{c}4 \\
(1,02)\end{array}$ & $\begin{array}{c}10 \\
(2,56)\end{array}$ & $\begin{array}{c}4 \\
(1,02)\end{array}$ & $\begin{array}{c}1 \\
(0,26)\end{array}$ & $\begin{array}{c}1 \\
(0,26)\end{array}$ \\
\hline Pascasarjana & $\begin{array}{c}2 \\
(0,51)\end{array}$ & $\begin{array}{c}2 \\
(0,51)\end{array}$ & $\begin{array}{c}0 \\
(0,00)\end{array}$ & $\begin{array}{c}2 \\
(0,51)\end{array}$ & $\begin{array}{c}0 \\
(0,00)\end{array}$ & $\begin{array}{c}0 \\
(0,00)\end{array}$ & $\begin{array}{c}0 \\
(0,00)\end{array}$ \\
\hline \multicolumn{8}{|l|}{ Profession } \\
\hline Housewife & $\begin{array}{c}231 \\
(59,08)\end{array}$ & $\begin{array}{c}104 \\
(26,60)\end{array}$ & $\begin{array}{c}127 \\
(32,48)\end{array}$ & $\begin{array}{c}84 \\
(21,48)\end{array}$ & $\begin{array}{c}75 \\
(19,18)\end{array}$ & $\begin{array}{c}53 \\
(13,55)\end{array}$ & $\begin{array}{c}19 \\
(4,86)\end{array}$ \\
\hline Farmer & $\begin{array}{c}56 \\
(14,32)\end{array}$ & $\begin{array}{c}27 \\
(6,91)\end{array}$ & $\begin{array}{c}29 \\
(0,74)\end{array}$ & $\begin{array}{c}14 \\
(3,58)\end{array}$ & $26(6,65)$ & $\begin{array}{c}11 \\
(2,81)\end{array}$ & $\begin{array}{c}5 \\
(1,28)\end{array}$ \\
\hline $\begin{array}{l}\text { Civil Servant } \\
\text { Retirement }\end{array}$ & $\begin{array}{c}28 \\
(7,16)\end{array}$ & $\begin{array}{c}21 \\
(5,37)\end{array}$ & $\begin{array}{c}7 \\
(1.79)\end{array}$ & $\begin{array}{c}19 \\
(4.86)\end{array}$ & $\begin{array}{c}6 \\
(1,53)\end{array}$ & $\begin{array}{c}2 \\
(0.51)\end{array}$ & $\begin{array}{c}1 \\
(0,26)\end{array}$ \\
\hline Swasta & $\begin{array}{c}23 \\
(5,88)\end{array}$ & $\begin{array}{c}9 \\
(2,30)\end{array}$ & $\begin{array}{c}14 \\
(3,58)\end{array}$ & $\begin{array}{c}11 \\
(2,81)\end{array}$ & $\begin{array}{c}7 \\
(1,79)\end{array}$ & $\begin{array}{c}5 \\
(1,28)\end{array}$ & $\begin{array}{c}0 \\
(0,00)\end{array}$ \\
\hline Wiraswata & $\begin{array}{c}26 \\
(6,65)\end{array}$ & $\begin{array}{c}13 \\
(3,32)\end{array}$ & $\begin{array}{c}13 \\
(3,32)\end{array}$ & $\begin{array}{c}8 \\
(2,05)\end{array}$ & $13(3,32)$ & $\begin{array}{c}4 \\
(1,02)\end{array}$ & $\begin{array}{c}1 \\
(0,26)\end{array}$ \\
\hline Does not work & $\begin{array}{c}27 \\
(6,91)\end{array}$ & $\begin{array}{c}12 \\
(3,07)\end{array}$ & $\begin{array}{c}15 \\
(3,84)\end{array}$ & $\begin{array}{c}7 \\
(1,79)\end{array}$ & $\begin{array}{c}7 \\
(1,79)\end{array}$ & $\begin{array}{c}9 \\
(2,30)\end{array}$ & $\begin{array}{c}4 \\
(1,02)\end{array}$ \\
\hline \multicolumn{8}{|c|}{ Income per month (rupiah) } \\
\hline$<3.000 .000$ & $\begin{array}{c}351 \\
(89,77)\end{array}$ & $\begin{array}{c}162 \\
(41,43)\end{array}$ & $\begin{array}{c}189 \\
(48,34)\end{array}$ & $\begin{array}{c}121 \\
(30,95)\end{array}$ & $\begin{array}{c}123 \\
(31,46)\end{array}$ & $\begin{array}{c}79 \\
(20,20)\end{array}$ & $\begin{array}{c}28 \\
(7,16)\end{array}$ \\
\hline$>3.000 .000$ & $\begin{array}{c}40 \\
(10,23)\end{array}$ & $\begin{array}{c}24 \\
(6,14)\end{array}$ & $\begin{array}{c}16 \\
(4,09)\end{array}$ & $\begin{array}{c}22 \\
(5,63)\end{array}$ & $11(2,81)$ & $\begin{array}{c}5 \\
(1,28)\end{array}$ & $\begin{array}{c}2 \\
(0,51)\end{array}$ \\
\hline \multicolumn{8}{|c|}{ Family history of hypertension } \\
\hline Yes & $\begin{array}{c}219 \\
(56,01)\end{array}$ & $\begin{array}{c}91 \\
(23,27)\end{array}$ & $\begin{array}{c}128 \\
(32,74)\end{array}$ & $\begin{array}{c}41 \\
(10,49)\end{array}$ & $\begin{array}{c}89 \\
(22,76)\end{array}$ & $\begin{array}{c}67 \\
(17,14)\end{array}$ & $\begin{array}{c}22 \\
(5,63)\end{array}$ \\
\hline None & $\begin{array}{c}172 \\
(43,99)\end{array}$ & $\begin{array}{c}95 \\
(24,30)\end{array}$ & $\begin{array}{c}77 \\
(19,69)\end{array}$ & $\begin{array}{c}102 \\
(26,09)\end{array}$ & $\begin{array}{c}45 \\
(11,51)\end{array}$ & $\begin{array}{c}17 \\
(4,35)\end{array}$ & $\begin{array}{c}8 \\
(2,05)\end{array}$ \\
\hline \multicolumn{8}{|l|}{ Body mass index } \\
\hline Underweight & $\begin{array}{c}37 \\
(9,46)\end{array}$ & $\begin{array}{c}18 \\
(4,60)\end{array}$ & $\begin{array}{c}19 \\
(4,86)\end{array}$ & $\begin{array}{c}14 \\
(3,58)\end{array}$ & $11(2,81)$ & $\begin{array}{c}10 \\
(2,56)\end{array}$ & $\begin{array}{c}2 \\
(0,51)\end{array}$ \\
\hline Normal & $\begin{array}{c}201 \\
(51,41)\end{array}$ & $\begin{array}{c}95 \\
(24,30)\end{array}$ & $\begin{array}{c}106 \\
(27,11)\end{array}$ & $\begin{array}{c}72 \\
(18,41)\end{array}$ & $\begin{array}{c}70 \\
(17,90)\end{array}$ & $\begin{array}{c}47 \\
(12,02)\end{array}$ & $\begin{array}{c}12 \\
(3,07)\end{array}$ \\
\hline Overweight & $\begin{array}{c}108 \\
(27,62)\end{array}$ & $\begin{array}{c}57 \\
(14,58)\end{array}$ & $\begin{array}{c}51 \\
(13,04)\end{array}$ & $\begin{array}{c}42 \\
(10,74)\end{array}$ & $37(9,46)$ & $\begin{array}{c}17 \\
(4,35)\end{array}$ & $\begin{array}{c}12 \\
(3,07)\end{array}$ \\
\hline Obese 1 & $\begin{array}{c}39 \\
(9,97)\end{array}$ & $\begin{array}{c}14 \\
(3,58)\end{array}$ & $\begin{array}{c}25 \\
(6,39)\end{array}$ & $\begin{array}{c}15 \\
(3,84)\end{array}$ & $13(3,32)$ & $\begin{array}{c}9 \\
(2,30)\end{array}$ & $\begin{array}{c}2 \\
(0,51)\end{array}$ \\
\hline Obese 2 & $\begin{array}{c}4 \\
(1,02)\end{array}$ & $\begin{array}{c}2 \\
(0,51)\end{array}$ & $\begin{array}{c}2 \\
(0,51)\end{array}$ & $\begin{array}{c}0 \\
(0,00)\end{array}$ & $\begin{array}{c}2 \\
(0,51)\end{array}$ & $\begin{array}{c}1 \\
(0,26)\end{array}$ & $\begin{array}{c}1 \\
(0,26)\end{array}$ \\
\hline Obese 3 & $\begin{array}{c}(1,02) \\
2 \\
(0,51)\end{array}$ & $\begin{array}{c}0 \\
(0,00)\end{array}$ & $\begin{array}{c}2 \\
(0,51)\end{array}$ & $\begin{array}{c}0 \\
(0,00)\end{array}$ & $\begin{array}{c}1 \\
(0,26)\end{array}$ & $\begin{array}{c}0 \\
(0,00)\end{array}$ & $\begin{array}{c}1 \\
(0,26)\end{array}$ \\
\hline
\end{tabular}




\begin{tabular}{|c|c|c|c|c|c|c|c|}
\hline \multirow[t]{2}{*}{ Characteristics } & \multirow[t]{2}{*}{ n (\%) } & \multicolumn{2}{|c|}{$\begin{array}{c}\text { Sleep Quality } \\
\text { n (\%) }\end{array}$} & \multicolumn{4}{|c|}{ Blood Pressure Value (\%) } \\
\hline & & Good & Poor & $\mathbf{N}$ & 1 & 2 & 3 \\
\hline \multicolumn{8}{|c|}{ History of habitual consumption of coffee, cigarettes and alcohol } \\
\hline No & $\begin{array}{c}274 \\
(70,08)\end{array}$ & $\begin{array}{c}131 \\
(33,50)\end{array}$ & $\begin{array}{c}143 \\
(36,57)\end{array}$ & $\begin{array}{c}101 \\
(25,83)\end{array}$ & $\begin{array}{c}94 \\
(24,04)\end{array}$ & $\begin{array}{c}60 \\
(15,35)\end{array}$ & $\begin{array}{c}19 \\
(4,86)\end{array}$ \\
\hline Smoking & $\begin{array}{c}5 \\
(1,28)\end{array}$ & $\begin{array}{c}2 \\
(0,51)\end{array}$ & $\begin{array}{c}3 \\
(0,77)\end{array}$ & $\begin{array}{c}1 \\
(0,26)\end{array}$ & $\begin{array}{c}2 \\
(0,51)\end{array}$ & $\begin{array}{c}0 \\
(0,00)\end{array}$ & $\begin{array}{c}2 \\
(0,51)\end{array}$ \\
\hline $\begin{array}{l}\text { Coffee } \\
\text { consumption }\end{array}$ & $\begin{array}{c}108 \\
(27,62)\end{array}$ & $\begin{array}{c}52 \\
(13,30)\end{array}$ & $\begin{array}{c}56 \\
(14,32)\end{array}$ & $\begin{array}{c}40 \\
(10,23)\end{array}$ & $36(9,21)$ & $\begin{array}{c}24 \\
(6,14)\end{array}$ & $\begin{array}{c}8 \\
(2,05)\end{array}$ \\
\hline $\begin{array}{l}\text { Smoking and } \\
\text { Coffee }\end{array}$ & $\begin{array}{c}4 \\
(1,02)\end{array}$ & $\begin{array}{c}1 \\
(0,26)\end{array}$ & $\begin{array}{c}3 \\
(0,77)\end{array}$ & $\begin{array}{c}1 \\
(0,26)\end{array}$ & $\begin{array}{c}2 \\
(0,51)\end{array}$ & $\begin{array}{c}0 \\
(0,00)\end{array}$ & $\begin{array}{c}1 \\
(0,26)\end{array}$ \\
\hline Alcohol & $\begin{array}{c}0 \\
(0,00)\end{array}$ & $\begin{array}{c}0 \\
(0,00)\end{array}$ & $\begin{array}{c}0 \\
(0,00)\end{array}$ & $\begin{array}{c}0 \\
(0,000)\end{array}$ & $\begin{array}{c}0 \\
(0,00)\end{array}$ & $\begin{array}{c}0 \\
(0,00)\end{array}$ & $\begin{array}{c}0 \\
(0,00)\end{array}$ \\
\hline \multicolumn{8}{|c|}{ Taking anti-hypertensive drugs } \\
\hline Yes & $\begin{array}{c}161 \\
(41,18)\end{array}$ & $\begin{array}{c}63 \\
(16,11)\end{array}$ & $\begin{array}{c}98 \\
(25,06)\end{array}$ & $\begin{array}{c}31 \\
(79,28)\end{array}$ & $\begin{array}{c}68 \\
(17,39)\end{array}$ & $\begin{array}{c}49 \\
(12,53)\end{array}$ & $\begin{array}{c}13 \\
(3,32)\end{array}$ \\
\hline No & $\begin{array}{c}230 \\
(58,82)\end{array}$ & $\begin{array}{c}123 \\
(31,46)\end{array}$ & $\begin{array}{c}107 \\
(27,37)\end{array}$ & $\begin{array}{c}112 \\
(28,64)\end{array}$ & $\begin{array}{c}66 \\
(16,88)\end{array}$ & $\begin{array}{c}35 \\
(8,95)\end{array}$ & $\begin{array}{c}17 \\
(4,35)\end{array}$ \\
\hline \multicolumn{8}{|c|}{ History of hypertension } \\
\hline Yes & $\begin{array}{c}219 \\
(56,01)\end{array}$ & $\begin{array}{c}129 \\
(32,99)\end{array}$ & $\begin{array}{c}106 \\
(27,11)\end{array}$ & $\begin{array}{c}121 \\
(30,95)\end{array}$ & $\begin{array}{c}69 \\
(17,65)\end{array}$ & $\begin{array}{c}38 \\
(9,72)\end{array}$ & $\begin{array}{c}7 \\
(1,79)\end{array}$ \\
\hline None & $\begin{array}{c}172 \\
(43,99)\end{array}$ & $\begin{array}{c}57 \\
(14,58)\end{array}$ & $\begin{array}{c}99 \\
(25,32)\end{array}$ & $\begin{array}{c}22 \\
(5,63)\end{array}$ & $\begin{array}{c}65 \\
(16,62)\end{array}$ & $\begin{array}{c}46 \\
(11,76)\end{array}$ & $\begin{array}{c}23 \\
(5,88)\end{array}$ \\
\hline \multicolumn{8}{|c|}{ Long time suffering from hypertension } \\
\hline None & $\begin{array}{c}225 \\
(57,54)\end{array}$ & $\begin{array}{c}126 \\
(32,23)\end{array}$ & $\begin{array}{c}99 \\
(25,32)\end{array}$ & $\begin{array}{c}115 \\
(29,41)\end{array}$ & $\begin{array}{c}66 \\
(16,88)\end{array}$ & $\begin{array}{c}35 \\
(8,95)\end{array}$ & $\begin{array}{c}9 \\
(2,30)\end{array}$ \\
\hline$<1$ y.o & $\begin{array}{c}27 \\
(6,91)\end{array}$ & $\begin{array}{c}10 \\
(2,56)\end{array}$ & $\begin{array}{c}17 \\
(4,35)\end{array}$ & $\begin{array}{c}6 \\
(1,53)\end{array}$ & $15(3,84)$ & $\begin{array}{c}4 \\
(1,02)\end{array}$ & $\begin{array}{c}2 \\
(0,51)\end{array}$ \\
\hline $1-4$ у.о & $\begin{array}{c}76 \\
(19,44)\end{array}$ & $\begin{array}{c}23 \\
(5,88)\end{array}$ & $\begin{array}{c}53 \\
(13,55)\end{array}$ & $\begin{array}{c}8 \\
(2,05)\end{array}$ & $35(8,95)$ & $\begin{array}{c}24 \\
(6,14)\end{array}$ & $\begin{array}{c}9 \\
(2,30)\end{array}$ \\
\hline $5-9$ y.o & $\begin{array}{c}45 \\
(11,51)\end{array}$ & $\begin{array}{c}17 \\
(4,35)\end{array}$ & $\begin{array}{c}28 \\
(7,16)\end{array}$ & $\begin{array}{c}10 \\
(2,56)\end{array}$ & $13(3,32)$ & $\begin{array}{c}14 \\
(3,58)\end{array}$ & $\begin{array}{c}8 \\
(2,05)\end{array}$ \\
\hline$\geq 10$ y.o & $\begin{array}{c}18 \\
(4,60)\end{array}$ & $\begin{array}{c}10 \\
(2,56)\end{array}$ & $\begin{array}{c}8 \\
(2,05)\end{array}$ & $\begin{array}{c}4 \\
(1,02)\end{array}$ & $\begin{array}{c}5 \\
(1,28)\end{array}$ & $\begin{array}{c}7 \\
(1,79) \\
\end{array}$ & $\begin{array}{c}2 \\
(0,51)\end{array}$ \\
\hline Total & 391 & 186 & 205 & 143 & 134 & 84 & 30 \\
\hline
\end{tabular}

Sleep quality has a significant correlation with blood pressure values in the elderly in Batu City. The higher the degree of blood pressure value, the more likely it is to have poor sleep quality. The elderly with normal blood pressure were 143 respondents (100.00\%), of which $71.33 \%$ had good sleep quality and $28.67 \%$ had poor sleep quality. The elderly with hypertension stage 1 were 134 respondents $(100.00 \%)$, of which $38.06 \%$ had good sleep quality and $61.94 \%$ had poor sleep quality. The elderly with hypertension stage 2 were 84 respondents (100.00\%), of which $28.57 \%$ had good sleep quality and $71.43 \%$ had poor sleep quality. The elderly with normal blood pressure were 30 respondents (100.00\%), of which $30.00 \%$ had good sleep quality and $70.00 \%$ had poor sleep quality. Cross-tabulation of sleep quality with the blood pressure value of the elderly in Batu City as shown in table 2.

Table 2 Cross Tabulation of Sleep Quality with Blood Pressure Values in Elderly

\begin{tabular}{|c|c|c|c|c|}
\hline \multirow[t]{2}{*}{ No. } & \multirow[t]{2}{*}{ Blood Pressure Value } & \multicolumn{2}{|c|}{ Sleep Quality } & \multirow[t]{2}{*}{ Total } \\
\hline & & Good & Poor & \\
\hline 1 & Normal & $102(71,33 \%)$ & $41(28,67 \%)$ & $143(100,00 \%)$ \\
\hline 2 & Hypertension stage 1 & $51(38,06 \%)$ & $83(61,94 \%)$ & $134(100,00 \%)$ \\
\hline 3 & Hypertension stage 2 & $24(28,57 \%)$ & $60(71,43 \%)$ & $84(100,00 \%)$ \\
\hline 4 & Hypertension stage 3 & $9(30,00 \%)$ & $21(70,00 \%)$ & $30(100,00 \%)$ \\
\hline & Total & 186 respondents & 205 respondents & 391 respondents \\
\hline
\end{tabular}

After doing bivariate analysis using Chi-square test, obtained p-value $<0.05$, which is equal to 0.00 so that $\mathrm{HO}$ is rejected and $\mathrm{H} 1$ is accepted. This means that there is a statistically significant correlation between blood pressure values and the sleep quality of the elderly in Batu City. 
Multivariate analysis was performed using the binary logistic regression analysis backward method. From the results of the regression analysis, the results of the Wald test with a sig of 0.000 showed that hypertension stage 1 influenced sleep quality. The OR obtained is 4.049 , which means that the chances of the elderly having poor sleep quality when experiencing hypertension stage 1 are 4.049 times compared to the elderly with normal blood pressure. In hypertension stage 2 , the chances of the elderly having poor sleep quality are 6.220 times compared to the elderly with normal blood pressure. Then the opportunity for the elderly to have poor sleep quality when experiencing hypertension stage 3 is 5.805 times compared to the elderly with normal blood pressure. Because OR $>1$, it can be concluded that hypertension stages 1, 2 and 3 increase the chances of poor sleep quality in the elderly in Batu City.

Table 3 Multivariate Analysis Results

\begin{tabular}{cccccccc}
\hline No & Variable & B & Wald & p value & OR & $\begin{array}{c}\text { CI } \\
\text { lower }\end{array}$ & $\begin{array}{c}\text { CI } \\
\text { upper }\end{array}$ \\
\hline $\mathbf{1}$ & Hypertension stage 1 & 1,398 & 29,697 & 0,000 & 4,049 & 2,448 & 6,695 \\
$\mathbf{2}$ & Hypertension stage 2 & 1,828 & 36,102 & 0,000 & 6,220 & 3,426 & 11,290 \\
$\mathbf{3}$ & Hypertension stage 3 & 1,759 & 16,032 & 0,000 & 5,805 & 2,454 & 13,730 \\
\hline
\end{tabular}

\section{Discussion}

The results of this study indicate that there is a significant correlation in sleep quality and the blood pressure value of the elderly in Batu City with the Chi-Square test obtained p-value 0.000 ( $p$ vale $<0.05$ ). It means that by improving the quality of sleep, the blood pressure value is also getting better (normal blood pressure value) (Table 2). So that sleep quality can be used as a predictor of success in hypertension therapy. On the other hand, sleep quality may affect blood pressure values, so efforts to improve sleep quality may improve blood pressure values.

This is following Lu's 2015 study which stated that hypertension was associated with an increase in poor sleep quality (10). The elderly who have good sleep quality tend to avoid hypertension and vice versa. The elderly who have poor sleep quality have the potential to experience hypertension. Several pathophysiological mechanisms support the changes in sympathetic nerve activity as evidenced by increased catecholamines and decreased heart rate (13). Lack of sleep duration can also increase the hemodynamic load, the sympathetic nervous system, continuous and prolonged exposure will result in vascular adaptation (14).

Poor sleep quality indicates a disturbance in the body's homeostasis. This is associated with an increased response of 2 axis, namely the Medulla Adrenal Sympathetic System and Hypothalamic Pituitary Adrenal-axis (HPA-axis) (15). The medulla adrenal can increase catecholamines. These catecholamines (norepinephrine and epinephrine) can cause effects on specific organs such as the heart and blood vessels in the form of vasoconstriction of peripheral blood vessels. This causes an increase in peripheral resistance (total peripheral resistance). Catecholamines also affect the contractility and conduction rate of myocytes which in turn causes an increase in cardiac output (16). Increased blood pressure occurs due to increased cardiac output and also total peripheral resistance (17).

Sleep also has a detectable modulating effect on HPA-axis activity. For someone with poor sleep quality, the pituitary will produce Corticotrophin Releasing Hormone (CRH) and Arginine Vasopressin (AVP). CRH secreted by the hypothalamus is then carried to the anterior pituitary which can then trigger corticotropin secretion, thereby increasing the hormone cortisol. So it can be said that lack of sleep and/or decreased sleep quality causes HPA-axis hyperactivation and an increase in the hormone cortisol (15). The action of cortisol causes an increase in the level of glucose in the bloodstream in two ways. First, by stimulating gluconeogenesis in the liver, the resulting glucose is released into the bloodstream and stored as glycogen. The second is by increasing glycogenolysis in the liver, thereby releasing large amounts of glucose into the bloodstream within minutes. Excess of gluconeogenesis and glycogenolysis causes hyperglycemia so that the blood becomes more viscous and then aggravate the work of the heart (18).

Vasopressin or ADH (antidiuretic hormone) can affect the kidneys' ability to reabsorb water. ADH induces the expression of water transport proteins in the distal tubule and collecting duct to increase water reabsorption which in turn increases plasma volume. This causes an increase in cardiac output so that blood pressure increases (19).

Sleep has important homeostatic functions, including suppressive effects on stress systems and proinflammatory systems. The stress response involves signalling the brain (hippocampus, amygdala and prefrontal cortex areas), the autonomic, cardiovascular and immune systems through neural and endocrine mechanisms. The inflammatory response induces endothelial dysfunction, plaque activation which further develops into atherosclerosis which can lead to hypertension (20). 
Sleep can affect the nervous system and organs function. Sleep also plays a role in the process of protein synthesis. Psychologically, sleep is very important, because people who have poor sleep quality tend to be irritable, lack concentration and have difficulty making decisions. Therefore, improving sleep quality is very important to maintain optimal blood pressure to avoid hypertension (14).

\section{Conclusion}

Based on the results and the data analysis of this study, it was concluded that there was a significant correlation between sleep quality and the blood pressure of the elderly in Batu City. Hypertension is associated with poor sleep quality, the chance of poor sleep quality increases in the elderly with hypertension in Batu City.

Based on the research that has been done, can be expressed suggestions for further research, hope that further research can be carried out to prove the effect of sleep quality on decreasing blood pressure values in the form of experimental tests. In addition, it is also necessary to continue to develop preventive and promotive efforts to obtain optimal blood pressure in hypertension patients to avoid complications or even death.

\section{Acknowledgements}

We would like to thank all respondents who participated in this research, as well as to all partners who helped to make this research well.

\section{References}

1. Liu R-Q, Qian Z, Trevathan E, Chang J-J, Zelicoff A, Hao Y-T, et al. Poor Sleep Quality Associated with High Risk of Hypertension and Elevated Blood Pressure in China: Results from A Large PopulationBased Study. Hypertens Res. 2016 Jan;39(1):54-9.

2. Kemenkes RI. Gambaran Kesehatan Lanjut Usia di Indonesia. Buletin Jendela Data dan Informasi Kesehatan. 2013;

3. Kemenkes RI. Pemeriksaan dan Pembinaan Kesehatan Haji Mencapai Istithaah Kesehatan Jemaah Haji untuk Menuju Keluarga Sehat (Petunjuk Teknis Permenkes Nomor 15 Tahun 2016). Jakarta; 2017.

4. Kemenkes RI. Hasil Utama Riskesdas 2018. Kemenkes RI Badan Penelitian dan Pengembangan Kesehatan. 2018;

5. Anorital. Morbiditas dan Multi Morbiditas Pada Kelompok Lanjut Usia di Indonesia. J Biotek Medisiana Indones. 2015;4, 77-88.

6. Widiana IMR, Ani LS. Prevalensi dan karakteristik hipertensi pada pralansia dan lansia di Dusun Tengah, Desa Ulakan, Kecamatan Manggis. E-J Med 6. 2017;

7. Danirmala D, Ariani P. Angka Kejadian Insomnia pada Lansia di Panti Tresna Werdha Wana Seraya Denpasar, Bali Tahun 2015. E-J Med 8. 2019;

8. Hanus JS, Amboni G, Rosa MI da, Ceretta LB, Tuon L. The quality and characteristics of sleep of hypertensive patients. Rev esc enferm USP. 2015 Aug;49(4):0596-602.

9. Tavasoli A, Saeidi M, Hooman N. Correlation Between Sleep Quality and Blood Pressure Changes in Iranian Children. J Compr Ped [Internet]. 2015 Feb 21 [cited 2019 Jun 16];6(1). Available from: http://comprped.neoscriber.org/en/articles/19815.html

10. Lu K, Chen J, Wu S, Chen J, Hu D. Interaction of Sleep Duration and Sleep Quality on Hypertension Prevalence in Adult Chinese Males. Journal of Epidemiology. 2015;25(6):415-22.

11. Mohani VS, Nurhayati T, Sari DM. Sleeping Quality Does Not Affect Blood Pressure among Elderly in Tresna Werdha Nursing Home City of Bandung. Journal of Medicine and Health [Internet]. 2018 Aug 31 [cited 2019 Jun 16];2(2). Available from: http://journal.maranatha.edu/index.php/jmh/article/view/1008

12. Sforza E, Saint Martin M, Barthelemy JC, Roche F. Association of Self-Reported Sleep and Hypertension in Non-Insomniac Elderly Subjects. JCSM [Internet]. 2014 Sep 15 [cited 2019 Dec 4]; Available from: http://jcsm.aasm.org/ViewAbstract.aspx?pid=29635

13. Guo J, Fei Y, Li J, Zhang L, Luo Q, Chen G. Gender- and age-specific associations between sleep duration and prevalent hypertension in middle-aged and elderly Chinese: a cross-sectional study from CHARLS 2011-2012. BMJ Open. 2016 Sep;6(9):e011770.

14. Martini S, Roshifanni S, Marzela F. Pola Tidur yang Buruk Meningkatkan Risiko Hipertensi. MKMI. 2018 Sep 20;14(3):297.

15. Minkel J, Moreta M, Muto J, Htaik O, Jones C, Basner M, et al. Sleep deprivation potentiates HPA axis stress reactivity in healthy adults. Health Psychology. 2014;33(11):1430-4.

16. Hakim F, Gozal D, Kheirandish-Gozal L. Sympathetic and Catecholaminergic Alterations in Sleep Apnea with Particular Emphasis on Children. Front Neur [Internet]. 2012 [cited 2019 Oct 6];3. Available from: http://journal.frontiersin.org/article/10.3389/fneur.2012.00007/abstract 
17. Park C, Fraser A, Howe LD, Jones S, Davey Smith G, Lawlor DA, et al. Elevated Blood Pressure in Adolescence Is Attributable to a Combination of Elevated Cardiac Output and Total Peripheral Resistance: Evidence Against a Hyperkinetic State. Hypertension. 2018 Nov;72(5):1103-8.

18. Goiato MC, da Silva EVF, Cândido NB, Nóbrega AS, de Medeiros RA, Sumida DH, et al. Evaluation of the level of cortisol, capillary blood glucose, and blood pressure in response to anxiety of patients rehabilitated with complete dentures. BMC Oral Health. 2019 Dec;19(1):75.

19. Cuzzo B, Lappin SL. Vasopressin (Antidiuretic Hormone, ADH) [Internet]. USA: StatPearls Publishing LLC; 2019. Available from: https://www.ncbi.nlm.nih.gov/books/NBK526069/

20. Palagini L, Maria Bruno R, Gemignani A, Baglioni C, Ghiadoni L, Riemann D. Sleep Loss and Hypertension: A Systematic Review. CPD. 2013 Mar 1;19(13):2409-19. 